\title{
Allyltrimethoxysilane as the Reagent of Double Bond Introduction to Porous Silica for Preparation of a Chiral Stationary Phase in Comparison with Allyltriethoxysilane
}

\author{
Won Jo Cheong, ${ }^{\star}$ You Jin Seo, Seong Tae Park, and Gyoung Won Kang \\ Department of Chemistry and Nano Fine Center, Inha University, Incheon 402-751, Korea. "E-mail: wicheong@inha.ac.kr \\ Received April 3, 2006
}

Key Words : Allyltrimethoxysilane, Chiral ligand, Improved carbon load, Cross polymerization

Chiral separation has been extensively used not only to produce pure enantiomers but also to analyze mixtures of enantiomers. Many different types of chiral stationary phases have been developed such as brush or ligand-exchange type phases, cyclodextrin phases, protein phases, polysaccharide phases, etc. Optically active polymers compose a great portion of the chiral phases and have been thoroughly investigated in a recent review. Among them, polysaccharide chiral phases have been known to be very useful for their versatility in chiral separation. ${ }^{2}$ Derivatives of polysaccharides adsorbed on porous silica particles are now very popular chiral stationary phases and are commercially available, but they are not compatible with some common $\mathrm{LC}$ solvents such as tetrahydrofuran and chloroform for their solubility in the solvents. ${ }^{3}$ Such a problem can be solved by securing chemical bonding between the silica surface and the polysaccharide ligand. ${ }^{2-5}$ Such phases (Diacel) have been commercially available only very recently. Introduction of double bonds to both the silica surface and the polysaccharide ligand followed by cross polymerization between the silica and the ligand has been the method of securing chemical bonding. The surface silanol groups of silica are used in the reaction of introducing double bonds, and two different reagents have been used to incorporate double bonds: 3-(trimethoxysilyl) propyl methacrylate ${ }^{5}$ and allyltriethoxysilane. . $^{\text {? }}$

Improvements in double bond introduction to the silica surface and consequent cross polymerization seem to be important for commercial polysaccharide chiral phases with chemically bonded polysaccharide ligands.

In this study, we have found that an improvement of the process can be achieved by simply employing allyltrimethoxysilane instead of allyltriethoxysilane(traditional reagent) as the reagent of double bond introduction.

\section{Experimental Section}

Chemicals. Heptane and 2-propanol were of HPLC grade and purchased from Fisher (Pittsburg, PA, USA) and used without purification. Allyltrimethoxysilane, allyltriethoxysilane, trans-stilbene oxide, benzoin, warfarin, 10-undecenoyl chloride, 3,5-dimethylphenylisocyanate and HMDS (1,1,1,3,3,3-hexamethyldisilazane) were purchased from Alldrich (Milwakee, IL, USA). AIBN ( $\alpha, \alpha$-azobisisobutyro-<smiles>C=CC[Si]12O[C@@H]3O[Si](CC=CC[Si](OC)(OC)OC)(OC3(C)O1)O2</smiles>

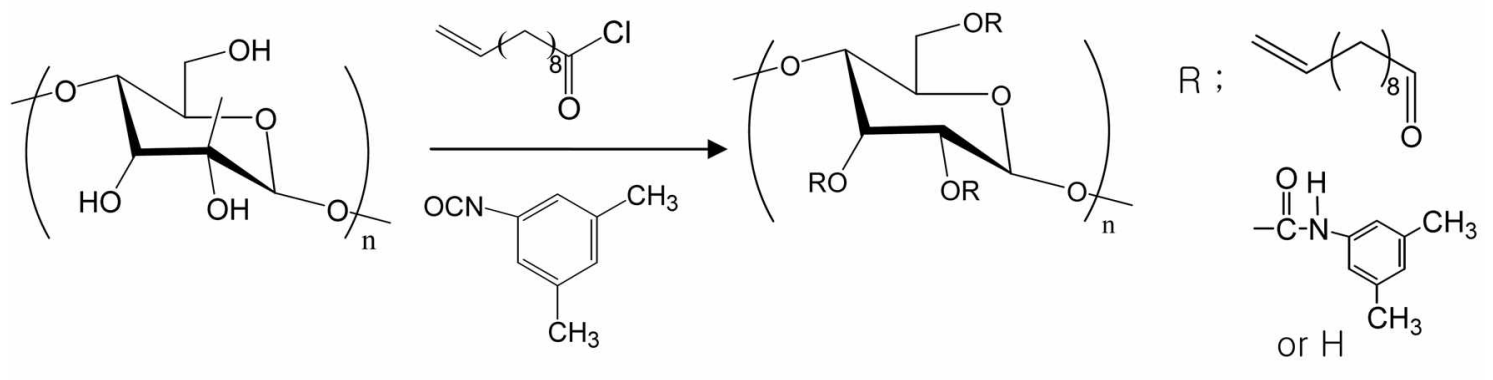

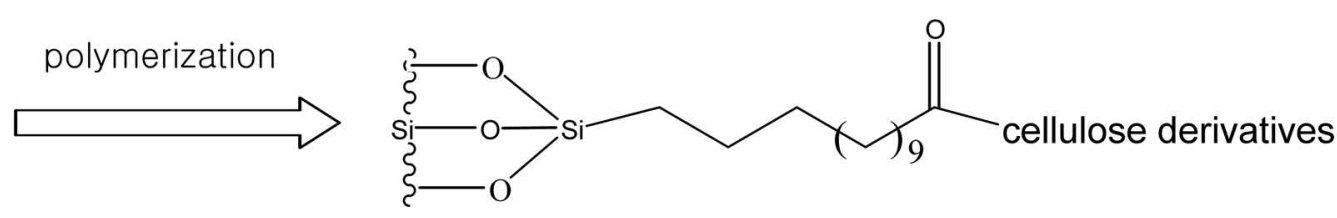

Figure 1. The overall schematic of synthesis of the chiral stationary phase by cross polyunerization of allylsilica with a cellulose derivative with double bonds. 
nitrile) was a gift from a neighboring laboratory. Microcrystalline cellulose was purchased form Merck (Whitehouse Station, NJ, USA). Silica gel (Adsorbosphere, $5 \mu, 80 \AA$ ) was obtained from Alltech (Deerfield, IL, USA).

HPLC. Two Shimadzu (Tokyo, Japan) I0AD pumps, a Shimadzu DGU-14A membrane degasser, a Rheodyne (Cotati, CA, USA) 7520 injector with a $0.5 \mu \mathrm{L}$ injection loop, an Isco (Lincoln, NE, USA) CV4 capillary window detector, and the home-made $0.5 \mathrm{~mm} \mathrm{ID}$ glass-lined microcolumn were combined to compose the HPLC system. The chromatographic data were obtained by a PC system, and a software Multichro 2000 from Youlin-Gisul (Sungnam, Korea) was used to acquire and process the data. An Alltech (Deerfield, USA) slurry packer was used to pack the microcolumns used. ${ }^{8}$

Synthesis of chiral stationary phase. The chiral stationary phases were prepared by cross polymerization of allylsilica with a cellulose derivative having double bonds (Figure 1).

The cellulose derivative was obtained by the process in the literature ${ }^{9}$ with some modifications. Microcrystalline cellulose $1 \mathrm{~g}, 10$-undecenoyl chloride $1 \mathrm{~g}$ were dissolved in pyridine and stirred for $1 \mathrm{hr}$ at $100^{\circ} \mathrm{C}$, then $1 \mathrm{~mL} 3,5-$ dimethylphenyl isocyanate was added and stirred for $24 \mathrm{hr}$ at $100^{\circ} \mathrm{C}$. Upon addition of methanol to the reaction mixture, the crude product was precipitated. The precipitate was dissolved in chloroform and recrystallized by methanol to give pure 10-undecenoyl-cellulose(3,5-dimethylcarbamate).

Porous allylsilica was prepared as follows. The porous silica $(5 \mu, 80 \AA)$ was baked at $300^{\circ} \mathrm{C}$ for $3 \mathrm{hr}$ to remove adsorbed water completely. The porous silica $1 \mathrm{~g}$ was dispersed in toluene and reacted with $0.7 \mathrm{~mL}$ allyltrimethoxysilane for $3.5 \mathrm{hr}$ at $110^{\circ} \mathrm{C}$ while stirring. Then, $0.4 \mathrm{~mL}$ HMDS was added for endcapping and the reaction mixture was stirred for $1 \mathrm{hr}$ at the same temperature. After completion of reaction, the silica was filtered, thoroughly washed with toluene, THF, $50 / 50(\mathrm{v} / \mathrm{v} \%)$ methanol/water, and methanol, and dried. Porous allylsilica was also prepared by the same procedure described above but using allyltriethoxysilane instead of allyltri-methoxy-silane for comparison purpose.

The allylsilica $1 \mathrm{~g}$ was dispersed in THF, and $0.30 \mathrm{~g}$ cellulose derivative and $0.015 \mathrm{~g}$ AIBN were added. The reaction mixture was evaporated in a rotavapor at room temperature. Cross polymerization was carried out at $100^{\circ} \mathrm{C}$ for $4 \mathrm{hr}$ while the bottle was rotated in the rotavapor. The final product was thoroughly washed with chloroform under reflux and acetone in series and dried to yield the chiral stationary phase.

The stationary phase (made by allyltrimethoxysilane vs. allyltriethoxysilane) was dispersed in methanol and packed in the microcolumn.

\section{Results and Discussion}

The results of chiral separation of R- and S- enantiomers of trans-stilbene oxide, benzoin, and warfarin by the column $(0.5 \times 300 \mathrm{~mm})$ packed with the chiral phase made by the conventional reagent (allyltriethoxysilane) are compared with the results obtained by the column packed with the chiral phase made by allyltrimethoxysilane in Figure 2 (for $90 / 10 \mathrm{v} / \mathrm{v} \%$ heptane $/ 2$-propanol), Figure 3 (for $98 / 2 \mathrm{v} / \mathrm{v} \%$ heptane/2-propanol), and Table 1 . The retention data of warfarin in 98/2 (v/v \%) heptane/2-propanol were not obtained since warfarin enantiomers retained too long in that eluent. The test samples were prepared by dissolving the solutes in pure 2-propanol since solubilities of the solutes in the eluents are limited. This discrepancy between the sample solvent and the eluent caused some strange looking system peaks in front of the main peaks of $\mathrm{R}$ - and S-enamtiomers, and they were confirmed by injection of 2-propanol and there was no confusion in identifying the main peaks.

We can easily note that the selectivities and resolutions obtained with the chiral phase made by allyltrimethoxy-
1) trans-stilbene oxide

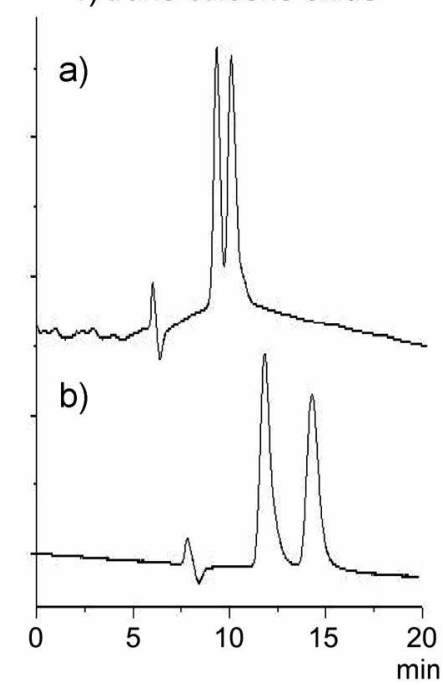

2) benzoin

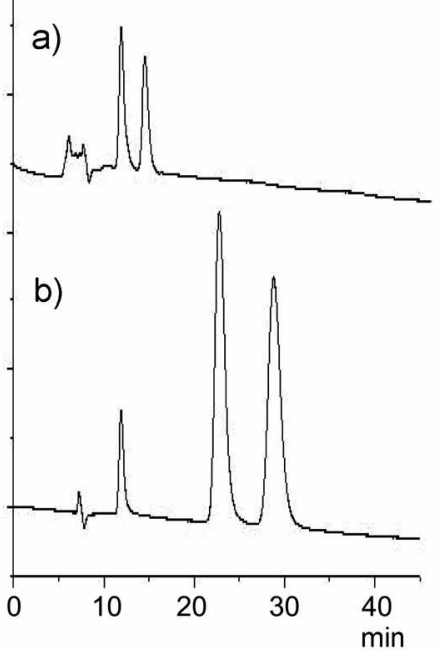

3) warfarin

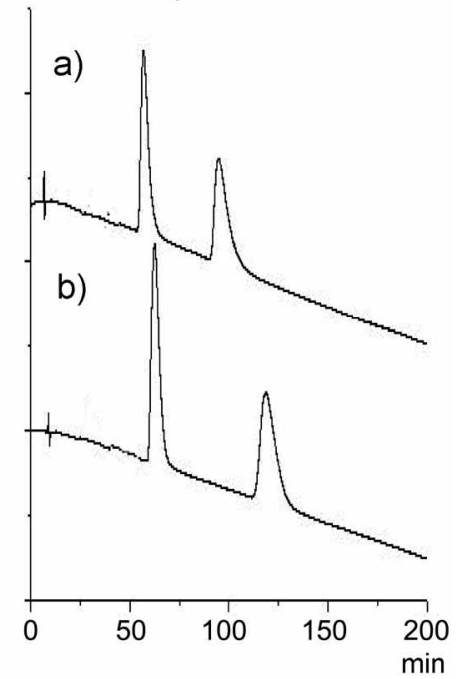

Figure 2. Comparison of Separation of R- and S- enantioners of trans-stilbene oxide, benzoin, and warfarin in $90 / 10$ (v/v \%) heptane/ 2-propanol at a flow rate of $10, \mathrm{~L} / \mathrm{min}$ by the column $(0.5 \times 300 \mathrm{~mm})$ packed with a) the chiral phase made by the conventional reagent (allyltriethoxysilane) and b) the chiral phase made by allyltrimethoxysilane. The detection wavelength was $254 \mathrm{~nm}$. 
1) trans-stilbene oxide

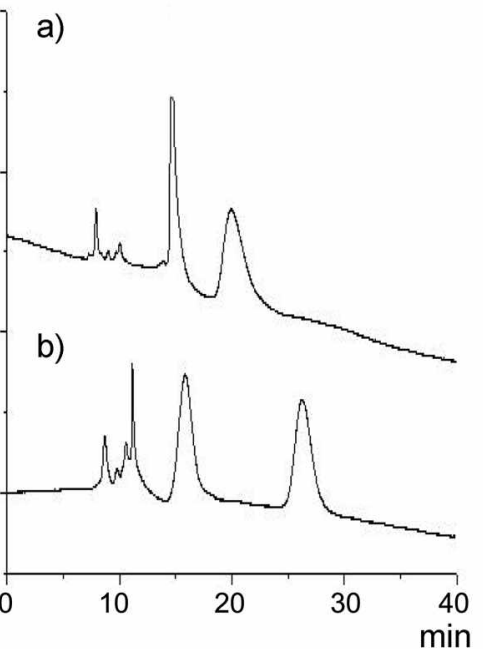

a)

2) benzoin

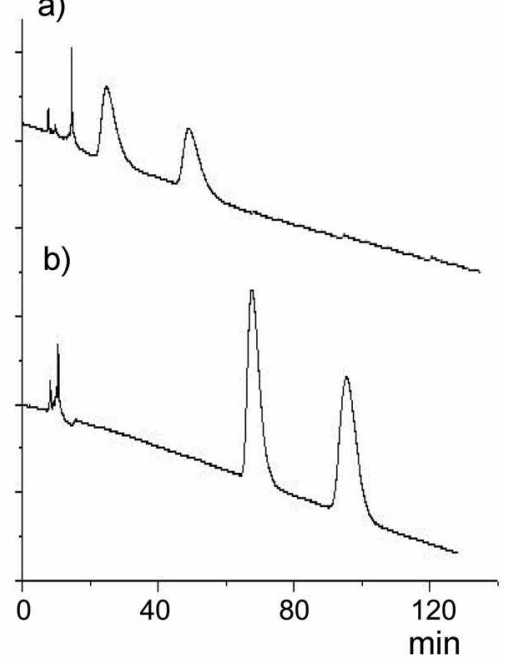

Figure 3. Comparison of Scparation of R- and S- enantioners of trans-stilbenc oxide and benzoin in $98 / 2$ (v/v $\%$ ) heptane/2-propanol at a flow rate of $10 \mu \mathrm{L} / \mathrm{min}$ by the column $(0.5 \times 300 \mathrm{~mm})$ packed with a) the chiral phase made by the conventional reagent (allyltriethoxysilane) and b) the chiral phase made by allyltrimethoxysilane. The detection wavelength was $254 \mathrm{~nm}$.

Table 1. Comparison of the selectivities (a) and resolutions(R) in separation of R- and S-cnantiomers of trans-stilbene oxide, benzoin, and warfarin by the chiral column $(0.5 \times 300 \mathrm{~mm})$ a) and b). a) The column packed with the chiral phase made by the conventional reagent (allyltriethoxysilane). b) The column packed with the chiral phase made by allyltrincthoxysilane

\begin{tabular}{|c|c|c|c|c|c|c|}
\hline \multirow[t]{2}{*}{ Elution condition } & \multicolumn{2}{|c|}{$\begin{array}{l}\text { trans-stilbene } \\
\text { oxide }\end{array}$} & \multicolumn{2}{|c|}{ benzoin } & \multicolumn{2}{|c|}{ wartarin } \\
\hline & $\alpha$ & Rs & $\alpha$ & Rs & $\alpha$ & Rs \\
\hline $90 / 10(v / v \%) \quad$ Column a) & 1.44 & 0.75 & 1.30 & 1.18 & 1.97 & 2.63 \\
\hline heptane/2-propanol Coluinn b) & 1.52 & 1.85 & 1.36 & 1.99 & 2.40 & 3.72 \\
\hline $98 / 2(\mathrm{v} / \mathrm{v} \%)$ & 1.66 & 2.06 & 1.78 & 2.64 & - & - \\
\hline heptane/2-propanol Column b) & 2.21 & 3.35 & 1.79 & 2.72 & - & - \\
\hline
\end{tabular}

silane were better than those obtained with the chiral phase made by allyltriethoxysilane although the extent of improvement was variant depending upon the solute types and elution conditions. This improvement in separation efficiency of allyltrimethoxysilane over allyltriethoxysilane was even more evident when a very short column was made for both cases and compared (Figure 4). We had to use a rather fast flow rate ( $50 \mu \mathrm{L} / \mathrm{min}$ ) for the microcolumn to observe the peaks of warfarin enantiomers since the retention times of warfarin isomers were too long even with the short column $(5 \mathrm{~cm})$ in $98 / 2(\mathrm{v} / \mathrm{v} \%)$ heptane/2-propanol. Anyway, in all cases, only one peak was observed for the chiral phase made by allyltriethoxysilane while two peaks (R- and Senantiomers) were observed for the chiral phase made by
1) trans-stilbene oxide a)

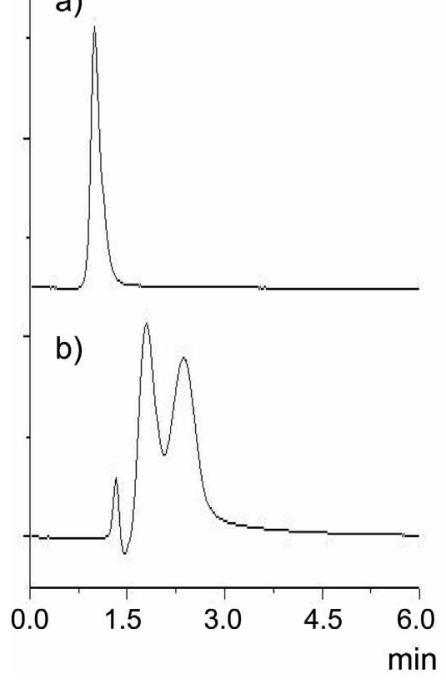

2) benzoin

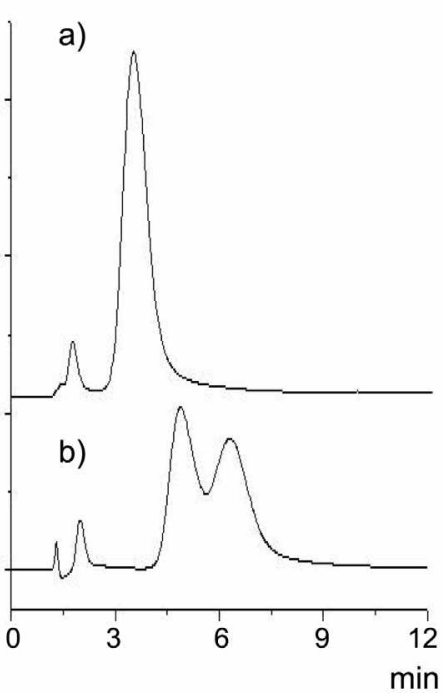

3) warfarin

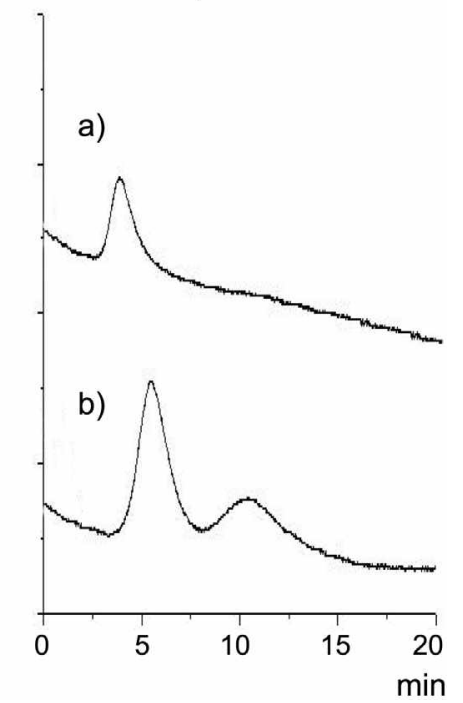

Figure 4. Comparison of Separation of R- and S- enantiomers of trans-stilbene oxide, benzoin, and warfarin in $98 / 2(\mathrm{v} / \mathrm{v} \%)$ heptane/ 2-propanol by the short column $(0.5 \times 50 \mathrm{~mm})$ packed with a) the chiral phase made by the conventional reagent (allyltriethoxysilane) and b) the chiral phase made by allyltrimethoxysilanc. The flow rate was $10 \mu \mathrm{L} / \mathrm{min}$ for trans-stilbene oxide and benzoin, and $50 \mu \mathrm{L} / \mathrm{min}$ for warfarin. The detection wavelength was $254 \mathrm{~nm}$. 
Table 2. The results of elemental analysis of silica treated with allytricthoxysilane vs. allyltrinethoxysilane and their reaction (cross polymerization) products with the polysaccharide derivative

\begin{tabular}{lccc}
\hline \multicolumn{1}{c}{ Description } & $\mathrm{C} \%$ & $\mathrm{H} \%$ \\
\hline $1 \quad \begin{array}{c}\text { Silica treated with allyltriethoxysilane } \\
\text { and end capped }\end{array}$ & 2.08 & 0.38 \\
$2 \quad$ Silica treated with allyltrimethoxysilane & 2.97 & 0.50 \\
$\quad$ and end capped & & \\
3 & 1 treated with the cellulose derivative & 6.30 & 1.09 \\
4 & 2 treated with the cellulose derivative & 10.84 & 1.65 \\
\hline
\end{tabular}

allyltrimethoxysilane.

Such superiority of the phase made with allyltrimethoxysilane over that made with allyltriethoxysilane was clear when the results of elemental analysis of both phases were compared (Table 2). We can note that more allyl groups were chemically attached to the silica by allyltrimethoxysilane than by allyltriethoxysilane and that consequently more polysaccharide ligands were chemically bound to the silica by cross polymerization. The retention times and separation efficiencies of a pair of enantiomers tend to improve as the content of the chiral ligand (carbon load) bound to the porous silica surface increases unless the ligand amount is larger than the limit over which the separation efficiency rather degrades because of too slow mass transfer rate. Actually, we observed longer retention times and better separation efficiencies with the chiral phase made by allyltrimethoxysilane than with the chiral phase made by allyltriethoxysilane (Figures 2-4).

The reason why an improved carbon load was observed when allyltrimethoxysilane is used instead of allyltri- ethoxysilane can be explained as follows. Methanol is supposed to be evolved when allyltrimethoxysilane reacts with a silanol group while ethanol should be evolved when allyltriethoxysilane is used. The reaction will proceed more readily if the reaction product (methanol or ethanol) is removed more easily. The boiling point of methanol is lower than that of ethanol and should be removed from the reaction mixture more readily. Furthermore, more bulky ethoxy groups will impose higher steric hindrance when an allyltriethoxysilane molecule approaches to a silanol group, which causes less reactivity of allyltriethoxysilane than allyltrimethoxysilane.

Acknowledgment. This work was supported by grant No. (R01-2006-000-10004-0) from the Basic Research Program of the Korea Science \& Engineering Foundation.

\section{References}

I. Yamamoto, C.; Okamoto, Y, Bull. Chem. Soc. Jpn, 2004, 77, 227,

2. Okamoto, Y,; Yashima, E. Angew. Chent. Int. Ed. 1998, 37, 1020.

3. Franco, P.; Senso, A.; Oliveros, L.; Minguillon, C. J. Chromatogr. A 2001, 906,155

4. Chen, X.; Liu, Y.; Qin, F.; Kong, L.; Zou, H. $J$. Chromatogr. $A$ $2003,1010,185$.

5. Chen, X.; Qin, F.; Liu, Y.; Huang, X.; Zou, H. J. Chromatogr: $A$ 2004, $1034,109$.

6. Minguillón, C.; Franco, P.; Oliveros, L. J. Chronatogr A 1996 , 728,415 .

7. Oliveros, L.; Senso, A.; Minguillón, C. Chirality 1997, 9, 145.

8. Ko, J. H.; Huang, H.; Kang, G. W.; Cheong, W. J. Bull. Konean Chem. Soc. 2005, 26, 1533.

9. Minguillón, C.; Franco, P.; Oliveros, L.; Lopez, P. J. Chromatogr. A $1996,728,407$ 